\title{
A mixed treatment comparison of selected osteopathic techniques used to treat acute nonspecific low back pain: a proof of concept and plan for further research
}

https://doi.org/10.1515/jom-2020-0268

Received October 14, 2020; accepted December 15, 2020; published online February 24, 2021

\begin{abstract}
Context: Back injuries have a high prevalence in the United States and can be costly for both patients and the healthcare system at large. While previous guidelines from the American College of Physicians for the management of acute nonspecific low back pain (ANLBP) have encouraged nonpharmacologic management, those treatment recommendations involved only superficial heat, massage, acupuncture, and spinal manipulation. Investigation about the efficacy of spinal manipulation in the management of ANLBP is warranted.
\end{abstract}

Objectives: To compare the results in previouslypublished literature documenting the outcomes of osteopathic manipulative treatment (OMT) techniques used to treat ANLBP. The secondary objective of this study was to demonstrate the utility of using Bayesian network metaanalysis (NMA) to perform a mixed treatment comparison (MTC) of a variety of osteopathic techniques.

Methods: A literature search for randomized controlled trials (RCTs) of ANLBP treatments was performed in April 2020 according to PRISMA guidelines by searching MEDLINE/PubMed, OVID, Cochrane Central, PEDro, and OSTMED.Dr databases; scanning the reference lists of articles; and using the Canadian Agency for Drugs and Technologies in Health grey literature checklist. Each database was searched from inception to April 1, 2020. The following search terms were used: acute low back pain, acute low back pain plus physical therapy, acute low back pain plus spinal manipulation, and acute low back pain plus osteopathic manipulation. The validity of eligible trials was

*Corresponding author: James W. Price, DO, MPH, Ascension St. Vincent Occupational Medicine Clinic, 14020 Old State Road, Evansville, IN 47725-1121, USA, E-mail: James.Price@ascension.org assessed by the single author using an adapted National Institute for Health and Care Excellence methodology checklist for randomized, controlled trials and an extraction form based on that checklist. The outcome measure chosen for this NMA was the Visual Analogue Scale of pain. The NMA were performed using the GeMTC user interface for automated NMA utilizing a Bayesian hierarchical model of random effects.

Results: The literature search initially found 483 unduplicated records. After screening and full text assessment, five RCTs were eligible for the MTC, yielding a total of 430 participants. Results of the MTC model suggested that there was no statistically significant decrease in reported pain when exercise, high-velocity low-amplitude (HVLA), counterstrain, muscle energy technique, or a mix of techniques were added to conventional treatment to treat ANLBP. However, the rank probabilities assessment determined that HVLA and the OMT mixed treatment protocol plus conventional care were ranked superior to conventional care alone for improving ANLBP.

Conclusions: While this study failed to provide definitive evidence upon which clinical recommendations can be based, it does demonstrate the utility of performing NMA for MTCs of osteopathic modalities used to treat ANLBP. However, to take full advantage of this statistical technique, future studies should be designed with consideration for the methodological shortcomings found in past osteopathic research.

Keywords: back injury; back pain; meta-analysis; OMT; osteopathic manipulative treatment.

Back injuries are the most costly and most prevalent disabling occupational injuries in the United States [1]. In 2016, musculoskeletal disorders involving the back accounted for $38.5 \%$ of all work-related musculoskeletal disorders [2]. In 2017, a systematic review and metaanalysis of randomized controlled trials [3] assessing the effectiveness of spinal manipulation therapy for the 
treatment of acute low back pain was published in the Journal of the American Medical Association [3]. In that study [3], 26 eligible randomized, controlled trials (RCTs) were identified. Fifteen of those RCTs provided moderatequality evidence that spinal manipulation had a statistically-significant association with improvements in pain, while 12 produced moderate-quality evidence that spinal manipulation therapy had a statistically-significant association with improvements in function [3].

The same year, the American College of Physicians (ACP) published a guideline presenting the evidence and clinical recommendations for noninvasive treatment of low back pain [4]. The guideline encouraged clinicians to inform all patients of the generally favorable prognosis of acute low back pain with or without sciatica, to advise patients to remain active, and to provide information about effective self-care options [4]. Nonsteroidal anti-inflammatory drugs or skeletal muscle relaxants were recommended if pharmacologic treatment is desired [4]. Recommended nonpharmacologic treatments in the guideline included superficial heat, massage, acupuncture, and spinal manipulation [4].

With spinal manipulation becoming a recommended nonpharmacological treatment for acute non-specific low back pain (ANLBP), a comparison of the effectiveness of different manual modalities is warranted. The primary objective of this study was to compare, in a systematic literature review and meta-analysis, the results of previous studies investigating the relative efficacy of several osteopathic manipulative treatment (OMT) techniques used to treat ANLBP. The secondary objective of this study was to demonstrate the utility of using Bayesian network metaanalysis (NMA) to perform a mixed treatment comparison (MTC) of a variety of osteopathic techniques.

\section{Methods}

A thorough literature search was performed in April 2020 according to Preferred Reporting Items for Systematic Reviews and Meta-Analyses (PRISMA) guidelines [5]. There were no outside sources of funding for this research project and institutional review board approval was not required.

Studies were identified by searching the MEDLINE/PubMed, OVID, Cochrane Central, PEDro, and OSTMED.Dr databases; scanning the reference lists of articles; and using the Canadian Agency for Drugs and Technologies in Health grey literature checklist [6]. Each database was searched from inception to April 1, 2020. The following search terms were used: acute low back pain, acute low back pain plus physical therapy, acute low back pain plus spinal manipulation, and acute low back pain plus osteopathic manipulation.

Articles studying outcomes of patients with ANLPB were selected to generate an MTC assessing pain related outcomes. To be eligible for the MTC, each study had to have been published in English; have been an RCT with human subjects; have had a population of working age (16-70 years); and had to have had an objective outcomes measure of pain assessed within one month from the date of onset.

Eligibility assessment was performed in an unblinded manner by the single author (J.W.P.) using the National Institute for Health and Care Excellence (NICE) methodology checklist for reviewing RCTs [7]. The NICE methodology checklist is a tool used to qualitatively assess RCTs for risk of four types of bias: selection bias, performance bias, attrition bias, and detection bias [7]. As there was only one reviewer, the author adapted the NICE checklist to add a quantifiable measure for determining suitability. Each category of bias was initially assigned a numerical value of one. One point was added for each 'no' or 'unclear' answer. The remaining value was the quality measure for the given type of potential bias (4=high risk; 3=moderate-high risk; $2=$ low-moderate risk, $1=$ low risk). The quality measures were summed and then divided by 4 . The average of the quality measures was then rounded to the nearest integer. Studies with an average value of three or four were eliminated from the NMA due to unacceptable risk of bias.

The author developed a data extraction sheet based on the NICE methodology checklist for reviewing RCT. Information was extracted from each study that qualified for full text assessment, including characteristics of trial participants (age and sex); type of intervention and comparison (placebo, nothing, or another intervention); type of outcomes measure; presented results; and bias assessment. The outcomes measure chosen for this NMA was the Visual Analogue Scale (VAS) instruments.

The NMA was performed using the GeMTC user interface for automated NMA utilizing a Bayesian hierarchical model of random effects $[8,9]$. Again, the primary outcome measure was mean VAS score. Heterogeneity priors were determined automatically by the software [10].

The network geometry is presented visually as a figure (Figure 1), with the treatment nodes representing the various treatments and comparisons from each of the included studies. The solid lines between some of the nodes indicate direct comparisons made between the intervention and control groups from the included RCTs. Indirect comparisons were made between nodes not connected by a solid line. Potential scale reduction factor (PSRF) was calculated to assess for adequate convergence within the network; PSRF compares the

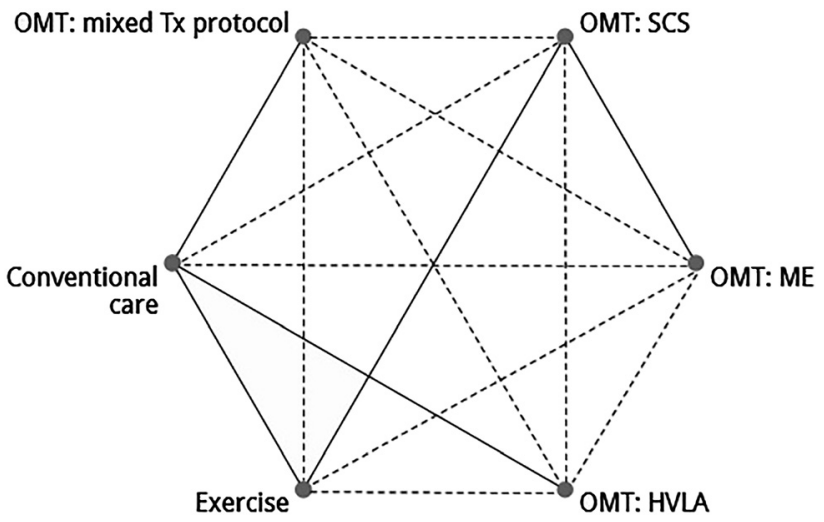

Figure 1: Presentation of the mixed treatment comparison network structure. The circles represent the interventions. The solid lines indicate direct comparisons between interventions. The dashed lines indicate indirect comparisons of interventions. OMT, osteopathic manipulative treatment; Tx, treatment; SCS, straincounterstrain; ME, muscle energy; HVLA, high-velocity lowamplitude. 
variation within each chain to the variation between chains. The PSRF starts with a high value, and slowly approaches 1.0 as the chains become more similar. Residual deviance $\left(D_{\text {res }}\right)$ was used to assess model fit. Model fit was deemed adequate when $D_{\text {res }}$ was equal to or less than the number of independent data points. Empirical examination for detecting across-studies (publication) bias was performed using a GeMTC produced comparison adjusted funnel plot of study effect.

Conventional care was selected as the baseline against which all other treatments were compared. Differences between treatments were considered significant (at the $5 \%$ level) if their confidence in tervals did not overlap the no-effect line. In addition to relative effects, the Bayesian analysis also generated a rank probabilities table.

\section{Results}

The literature search identified 805 studies meeting search criteria. After removing duplicates, 483 studies remained to be screened for eligibility (Figure 2). The screening excluded 469 studies. The full text of the remaining 14 studies [11-24] were assessed by the single author (J.W.P.) for risk of bias. The results of the risk of bias assessment are presented in Table 1. Seven studies [12, 15, 18, 20, 22-24] failed to present the numerical values, confidence intervals, or standard deviations of their findings. High overall risk of bias was identified for two of the remaining studies [11, 14]. High risk of performance bias was determined for one study [14], and high risk of selection bias was suspected for one study [20]. This left five studies [13, 16, 17, $19,21]$ to be used for the MTC model. The characteristics of each, including interventions, are presented in Table 2. Each of the groups in the included studies had small sizes, ranging from 30 to 70 subjects. The OMT groups were particularly small, with 30-44 subjects. High-velocity lowamplitude technique (HVLA), strain-counterstrain technique (SCS), muscle energy technique (MET), and a mixed OMT protocol were included in the model. It is also worth noting that the MET and SCS interventions were limited to treating the erector spinae muscle group.

The network geometry of the resulting model consisted of six nodes, five direct comparisons, and no closed loops (Figure 1). The exercise node had a sample size of 118 patients from two studies $[16,17]$. The counterstrain node had 74 subjects from two studies $[16,19]$. The mixed treatment protocol node had 33 subjects from a single trial [13]. The

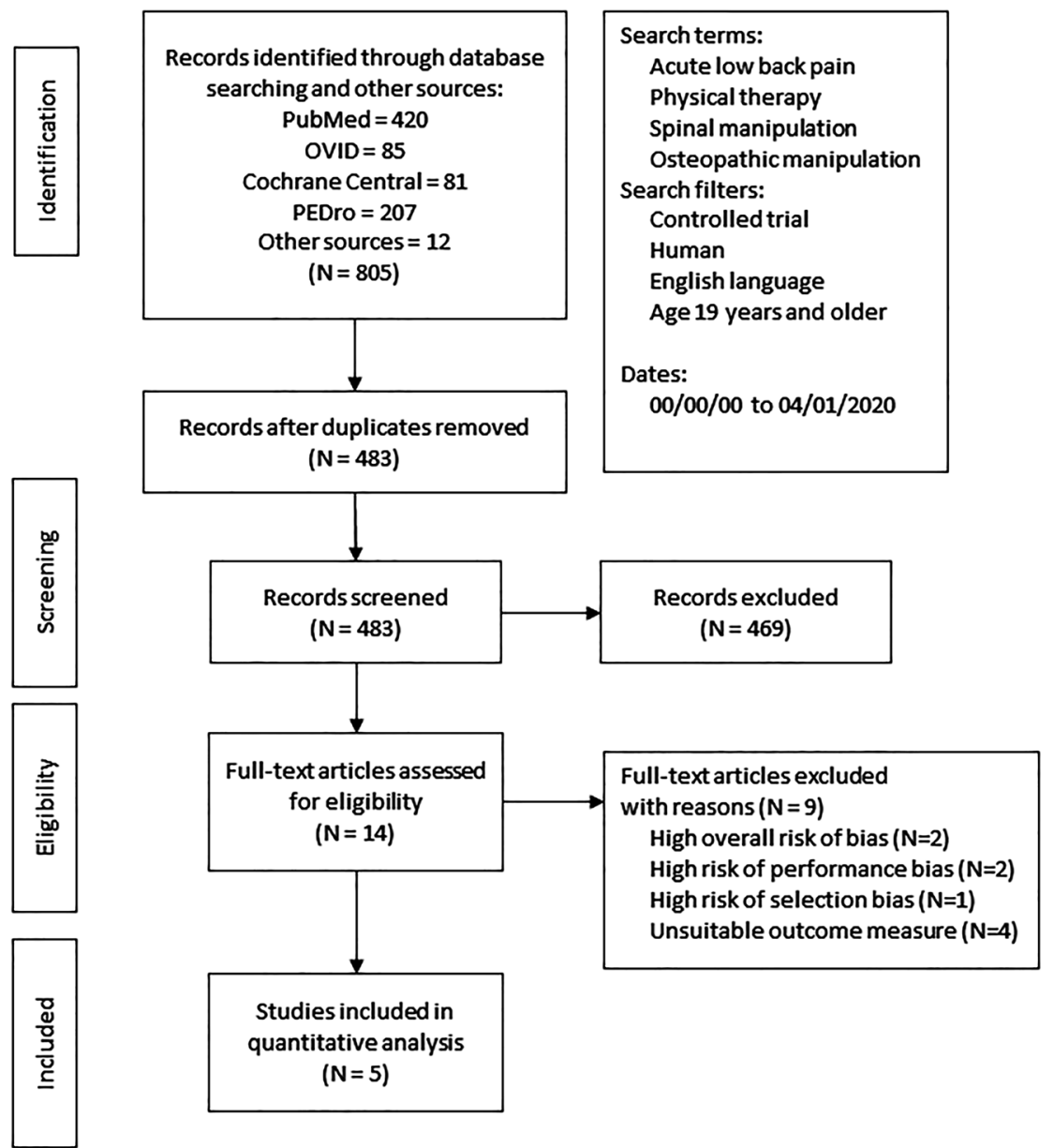

Figure 2: Preferred Reporting Items for Systematic Reviews and Metaanalyses (PRISMA) literature search flow diagram. 
Table 1: Risk of bias according to National Institute for Health and Care Excellence checklist for randomized controlled trials.

\begin{tabular}{|c|c|c|c|c|c|c|}
\hline Lead Author, Year & $\begin{array}{l}\text { Selection } \\
\text { bias }\end{array}$ & $\begin{array}{l}\text { Performance } \\
\text { bias }\end{array}$ & $\begin{array}{l}\text { Attrition } \\
\text { bias }\end{array}$ & $\begin{array}{l}\text { Detection } \\
\text { bias }\end{array}$ & $\begin{array}{l}\text { Overall risk } \\
\text { of bias }\end{array}$ & $\begin{array}{c}\text { Unsuitable outcome } \\
\text { measure }\end{array}$ \\
\hline Ali (2015) [11] & 1 & 3 & 3 & 3 & 3 & \\
\hline Blomberg (1992) [12] & 2 & 4 & 3 & 3 & 3 & $x$ \\
\hline Cruser (2012) [13] & 1 & 3 & 1 & 1 & 2 & \\
\hline Goertz (2013) [14] & 2 & 4 & 2 & 2 & 3 & \\
\hline Hurley (2004) [15] & 1 & 3 & 1 & 2 & 2 & $\mathrm{x}$ \\
\hline Lewis (2011) [16] & 1 & 3 & 1 & 3 & 2 & \\
\hline Machado (2010) [17] & 1 & 3 & 1 & 1 & 2 & \\
\hline Paatelma (2008) [18] & 1 & 3 & 2 & 1 & 2 & $\mathrm{X}$ \\
\hline Prashant (2010) [19] & 1 & 3 & 1 & 3 & 2 & \\
\hline Saratchandran (2013) [20] & 4 & 3 & 1 & 3 & 3 & $\mathrm{x}$ \\
\hline Schneider (2016) [21] & 1 & 2 & 1 & 1 & 1 & \\
\hline Takamoto (2015) [22] & 2 & 2 & 2 & 1 & 2 & $\mathrm{x}$ \\
\hline Vohra (2014) [23] & 4 & 4 & 4 & 4 & 4 & $\mathrm{x}$ \\
\hline von Heymann (2013) [24] & 1 & 2 & 2 & 1 & 2 & $x$ \\
\hline Low risk of bias & 1 & \multicolumn{3}{|c|}{ Low risk of bias } & 1 & \\
\hline Low to moderate risk of bias & 2 & \multicolumn{3}{|c|}{ Moderate risk of bias } & 2 & \\
\hline Moderate to high risk of bias & 3 & \multicolumn{3}{|c|}{ High risk of bias } & 3 & \\
\hline High risk of bias & 4 & \multicolumn{3}{|c|}{ Very high risk of bias } & 4 & \\
\hline
\end{tabular}

HVLA node had 37 subjects from only one study [21], and the muscle energy node contained 30 subjects, also from a single study [19]. As such, the model relied heavily on indirect comparisons. The PSRF compares the variation within each chain to the variation between chains. The PSRF was below 1.05 for each of the comparisons made in this model, indicating adequate convergence of the model (Table 3). The overall $D_{\text {res }}$ was 10 , with 10 data points; this is consistent with adequate model fit. The per-arm residual deviance for each of the included studies determined that there were no important outliers (Table 4).

Table 5 presents comparisons of the studied interventions with mean difference and $95 \%$ confidence intervals (CI). Each cell in the league table represents the effect of column-defining intervention relative to the row defining intervention. The $95 \% \mathrm{CI}$ for each comparison crossed zero, indicating that there were no statistically significant mean differences. The relative effects plot (Figure 3) graphically presents the VAS mean difference and $95 \% \mathrm{CI}$ for each active treatment relative to conventional care, suggesting that there was no statistically significant decrease of reported pain when exercise or OMT were added to conventional treatment. However, the highvelocity low-amplitude (HVLA) arm and OMT mixed treatment protocol arm trended toward demonstrating an added benefit. Inspection of rank probabilities (Table 6) demonstrated that HVLA and OMT mixed treatment protocols plus conventional care were ranked superior to conventional care alone for improving ANLBP (Table 6).

Heterogeneity was not a consideration for this model because each direct comparison in the network had only a single study; inconsistency was not assessed because the model had no closed loops [25]. The comparison adjusted funnel plot of study effect detected no indication of acrossstudies bias (Figure 4).

\section{Discussion}

The findings of this systematic review and meta-analysis are not conclusive, but they suggest that the HVLA and OMT mixed treatment protocol arms in prior studies trended toward being superior to erecter spinae muscle energy (MET) and strain-counterstrain (SCS) techniques for reducing ANLBP in the first month after onset. These findings also suggest that the addition of prescribed exercises offered no therapeutic advantage over conventional treatment alone.

The results of this MTC should not be used to infer that MET and SCS are ineffective techniques for ANLBP. However, the findings do suggest that MET and SCS directed at erector spinae dysfunction trended toward being inferior to 


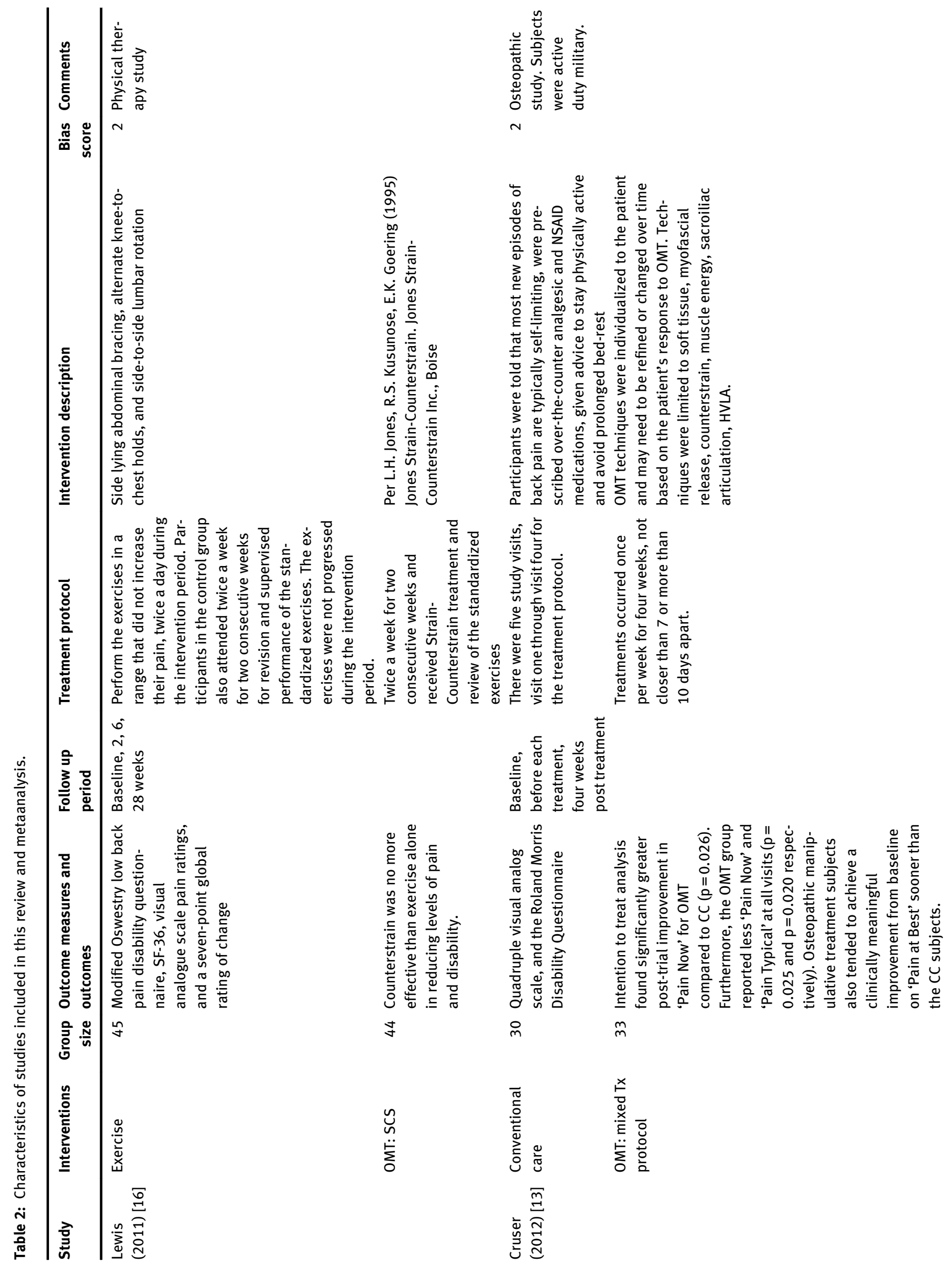




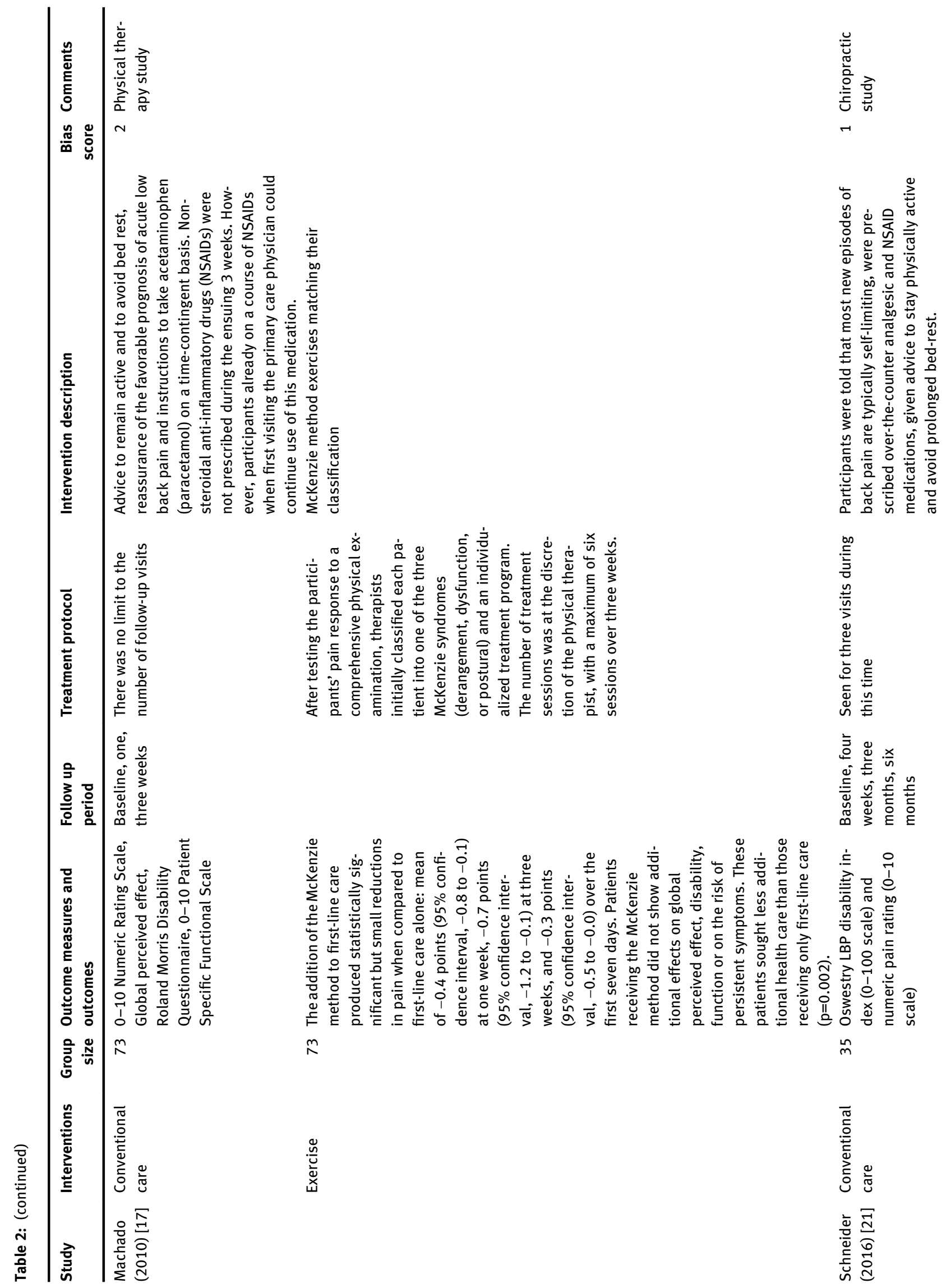




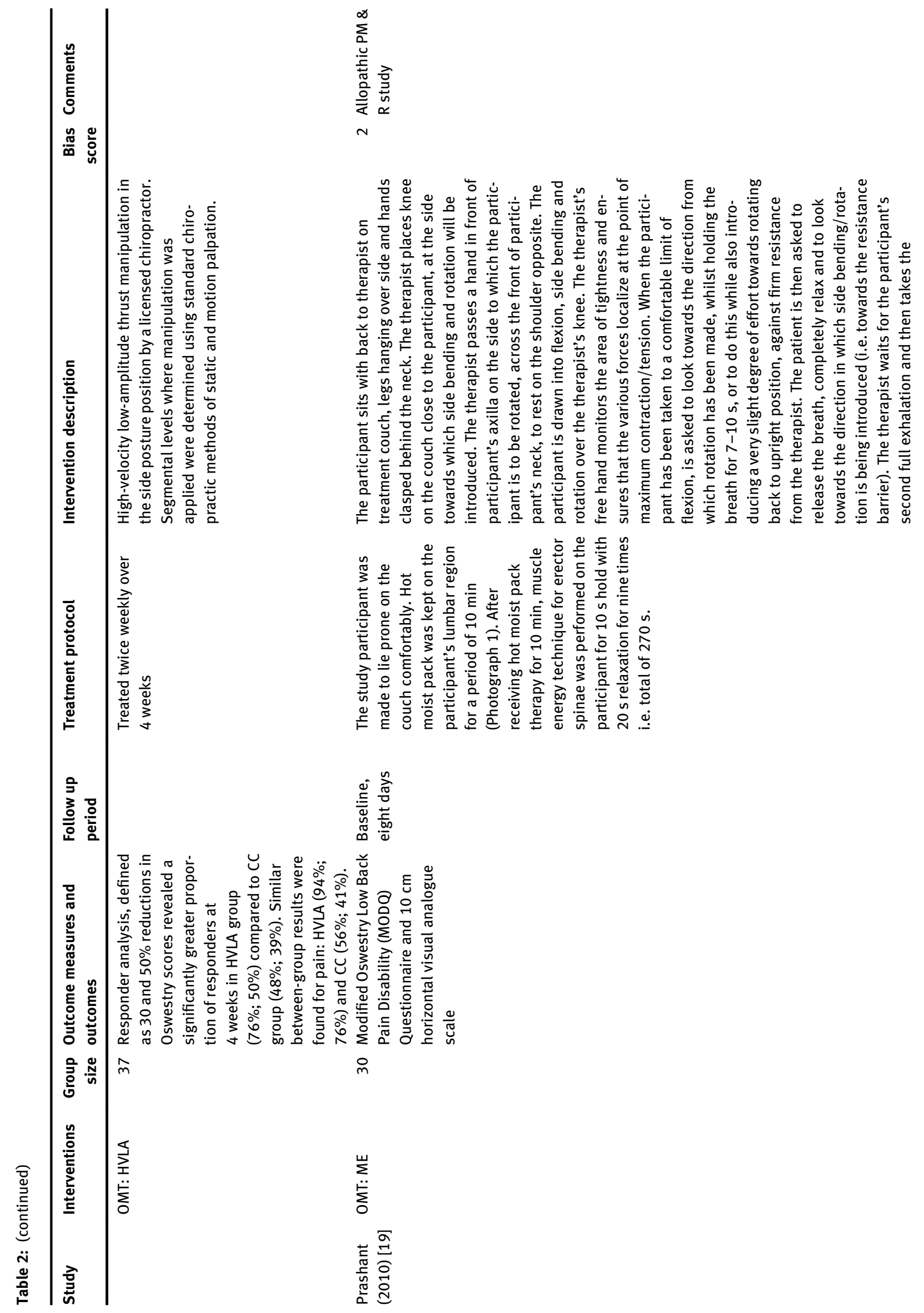


Table 3: Per-parameter convergence diagnostics.

\begin{tabular}{lcrrr}
\hline Parameter & $\begin{array}{r}\text { Standard } \\
\text { deviation }\end{array}$ & $\begin{array}{r}\text { Time-series } \\
\text { SE }\end{array}$ & & \multicolumn{2}{c}{$\begin{array}{r}\text { Potential scale } \\
\text { reduction factor, } \\
\text { PSRF }\end{array}$} \\
\cline { 3 - 5 } & & & $\begin{array}{r}\text { Point } \\
\text { estimate }\end{array}$ & $\begin{array}{r}97.5 \% \\
\text { quantile }\end{array}$ \\
\hline $\begin{array}{l}\text { Conventional care, } \\
\text { OMT: HVLA }\end{array}$ & 1.1931 & 0.013608 & 0.99975 & 0.99991 \\
$\begin{array}{l}\text { Conventional care, } \\
\begin{array}{l}\text { OMT: mixed tx } \\
\text { protocol }\end{array}\end{array}$ & 1.207 & 0.014049 & 1.0011 & 1.0036 \\
$\begin{array}{l}\text { Exercise, Conven- } \\
\text { tional care }\end{array}$ & 1.4552 & 0.017588 & 1.001 & 1.0039 \\
$\begin{array}{l}\text { Exercise, OMT: } \\
\begin{array}{l}\text { SCS } \\
\text { OMT: SCS, OMT: }\end{array}\end{array}$ & 1.1557 & 0.013394 & 1.0004 & 1.0011 \\
$\begin{array}{l}\text { ME } \\
\begin{array}{l}\text { Random effects } \\
\text { standard deviation }\end{array}\end{array}$ & 0.54666 & 0.0093508 & 1.0004 & 1.0019 \\
\hline
\end{tabular}

HVLA, high-velocity low-amplitude; ME, muscle energy;

OMT, osteopathic manipulative treatment; SCS, strain-counterstrain; $\mathrm{SE}$, standard error.

Table 4: Residual deviance per arm, per study.

\begin{tabular}{llrr}
\hline Study & Arm & $\begin{array}{r}\text { Residual } \\
\text { deviance }\end{array}$ & Leverage \\
\hline Lewis (2011) & Exercise & 0.987 & 0.987 \\
& OMT: SCS & 1.001 & 1.001 \\
Cruser (2012) & Conventional care & 1.037 & 1.035 \\
& OMT: mixed Tx protocol & 1.010 & 1.010 \\
Machado (2010) & Conventional care & 0.988 & 0.988 \\
& Exercise & 0.991 & 0.991 \\
Schneider (2016) & Conventional care & 0.987 & 0.987 \\
& OMT: HVLA & 0.987 & 0.987 \\
Prashant (2010) & OMT: ME & 0.979 & 0.978 \\
& OMT: SCS & 1.017 & 1.017 \\
\hline
\end{tabular}

HVLA, high-velocity low-amplitude; ME, muscle energy; OMT, osteopathic manipulative treatment; ROM, range of motion; SCS, strain-counterstrain; Tx, treatment.

conventional care alone. This may be related to the effectiveness of the studied techniques, or erector spinae dysfunction may not be a major contributor to ANLBP.

These findings agree with two previous Cochrane reviews, one examining the use of muscle energy technique for low back pain [26] and one exploring the effect of prescribed exercises for low back pain [27]. A 2016 Cochrane review [26] determined that there was low-quality evidence of no clinically relevant difference from adding MET to other interventions for management ANLBP with the outcome of pain. There was low-quality evidence of an effect in favor of MET for functional status. However, the 
Table 5: Comparison of the included interventions: mean difference $(95 \% \mathrm{Cl})$.

$\begin{array}{llllll}\text { Conventional care } & 0.093(-2.792,2.965) & -1.902(-4.445,0.593) & 0.496(-3.939,4.944) & 0.301(-3.501,4.165) & -1.740(-4.273,0.778) \\ & \text { Exercise } & -2.007(-5.767,1.849) & 0.390(-3.025,3.797) & 0.204(-2.242,2.714) & -1.821(-5.643,1.922) \\ & & \text { OMT: HVLA } & 2.414(-2.804,7.512) & 2.193(-2.356,6.748) & 0.161(-3.394,3.704) \\ & & \text { OMT: ME } & -0.190(-2.527,2.204) & -2.214(-7.219,2.763) \\ & & & \text { OMT: SCS } & -2.024(-6.627,2.438) \\ & & & & \text { OMT: mixed Tx protocol }\end{array}$

Comparison of the included interventions: mean difference $(95 \% \mathrm{Cl})$. Each cell gives the effect of the column-defining intervention relative to the row-defining intervention. $\mathrm{Cl}$, confidence interval; HVLA, high-velocity low-amplitude; ME, muscle energy; OMT, osteopathic manipulative treatment; ROM, range of motion; SCS, strain-counterstrain; Tx, treatment

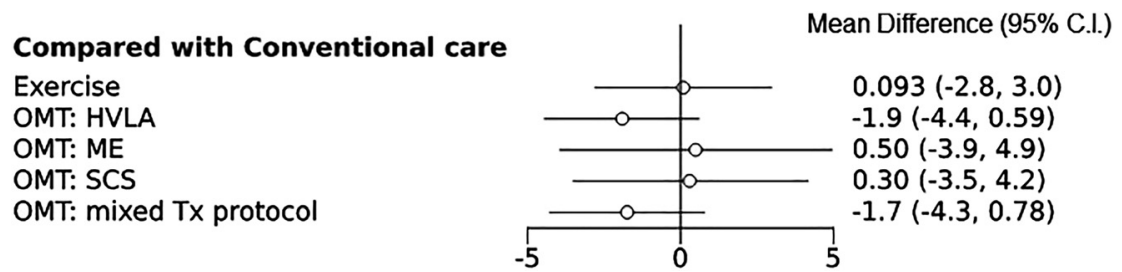

Figure 3: Relative effects plot. OMT, osteopathic manipulative treatment; Tx, treatment; SCS, strain-counterstrain; ME, muscle energy; HVLA, high-velocity low-amplitude.

Table 6: Rank probabilities.

\begin{tabular}{lrrrrrr}
\hline & Rank & Rank & Rank & Rank & Rank & Rank \\
& $\mathbf{1}$ & $\mathbf{2}$ & $\mathbf{3}$ & $\mathbf{4}$ & $\mathbf{5}$ & $\mathbf{6}$ \\
\hline Conventional care & 0.003 & 0.040 & $\mathbf{0 . 3 9 7}$ & 0.159 & 0.149 & 0.252 \\
Exercise & 0.029 & 0.081 & 0.203 & $\mathbf{0 . 3 5 4}$ & 0.195 & 0.139 \\
OMT: HVLA & $\mathbf{0 . 4 8 6}$ & 0.326 & 0.068 & 0.055 & 0.043 & 0.023 \\
OMT: ME & 0.058 & 0.070 & 0.115 & 0.150 & 0.214 & $\mathbf{0 . 3 9 4}$ \\
OMT: SCS & 0.039 & 0.079 & 0.139 & 0.227 & $\mathbf{0 . 3 5 0}$ & 0.166 \\
OMT: mixed Tx & 0.385 & $\mathbf{0 . 4 0 5}$ & 0.079 & 0.055 & 0.050 & 0.026 \\
protocol & & & & & & \\
\hline
\end{tabular}

Bold values indicate statistical significance. HVLA, high-velocity low-amplitude; ME, muscle energy; OMT, osteopathic manipulative treatment; ROM, range of motion; SCS, strain-counterstrain; Tx, treatment.

authors also noted that the quality of research related to testing the effectiveness of MET was poor [26]. The 12 studies in the review were generally small $(n=20-72)$ and at high risk of bias due to methodological inadequacies; there was not sufficient evidence to reliably determine whether MET was likely to be effective in practice [26]. The authors of that review [26] also stated that there is a need for larger, higher-quality studies with more robust methodology. Ideally, all studies should clearly describe all methods, have larger sample sizes, use robust methods of statistical analysis, demonstrate baseline equivalence of patient characteristics between groups, and use treatment protocols that can be generalized to clinical practice. A 2005 Cochrane review [27] of 61 randomized controlled trials with a total of 6,390 participants concluded that exercise

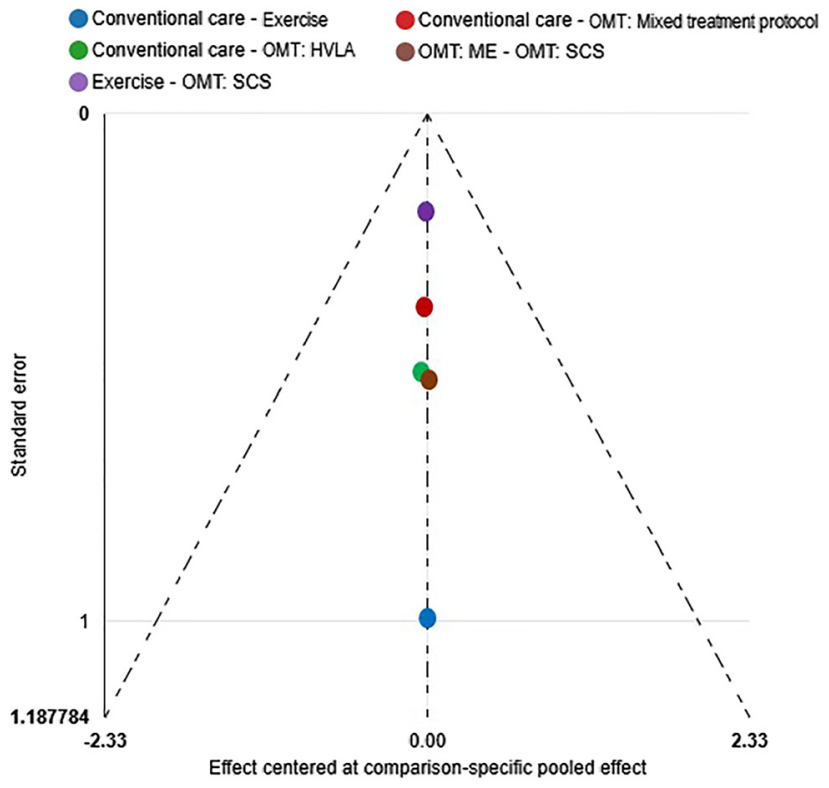

Figure 4: Funnel plot for detecting across-studies bias. OMT, osteopathic manipulative treatment; Tx, treatment; SCS, straincounterstrain; ME, muscle energy; HVLA, high-velocity lowamplitude.

therapy was as effective as either no treatment or other conservative treatments for ANLBP. However, the authors also noted that their review largely reflected limitations of the literature, including low quality studies with heterogeneous outcomes measures, inconsistent and poor reporting, and possibility of publication bias [27]. 
The model presented in this study is subject to the limitations noted by the authors of the previous Cochrane reviews [26, 27]. Heterogenous study methodologies and outcome measures were identified during the literature review. This resulted in a limited number of studies meeting inclusion criteria. Because of this, exercise was included as a variable to allow the inclusion of muscle energy technique and counterstrain in the final model. Fortunately, the use of exercise for acute LBP has been researched extensively, thus providing a point of reference from which to compare the results of the exercise arm of this MTC to evaluate the utility of this methodology. The sample sizes for each of the studies included in the model were small and likely contributed to the failure to demonstrate any statistically significant results (Table 2). Several of the included studies had moderate to high risk of performance bias because of failing to blind participants to their allocation group and lack of clarity as to whether comparison groups received the same care and support apart from the intervention studied (Table 1). A few of the included studies had moderate to high risk for detection bias for failing to blind investigators to the participants' intervention and to important confounding factors.

A limitation that is unique to the present review was having a single author (J.W.P.) who performed the literature search and quality assessment. This may have led to the introduction of selection bias. An attempt was made to mitigate this issue by modifying the NICE checklist to generate a more objective quantitative measure of bias risk.

While this study failed to provide any definitive evidence upon which to make clinical recommendations, it did demonstrate the utility of performing NMA for MTCs of osteopathic modalities used to treat ANLBP. However, to take full advantage of this statistical technique, future studies should be designed with consideration for the shortcomings in osteopathic research identified by this study and noted in the previous Cochrane reviews [26, 27].

To address these deficiencies, the author proposes a coordinated series of RCTs with an identical design other than the studied intervention. Individual studies would be performed at multiple sites coordinated through an interinstitutional research network. The intervention would be a single OMT technique plus conventional care or a mixed treatment protocol plus conventional care. The chosen intervention would be used to treat all identified dysfunctions. The control could be a different OMT technique plus conventional care for all identified dysfunctions, sham OMT plus conventional care, or only conventional care. The choice of the intervention and the control would be left to the institution performing the study. Inclusion criteria for participants in the series suggested by the author here would be adults who meet the case criteria for ANLBP. ANLPB should be uniformly defined. A suggested definition is pain from the lower costal margin to the gluteal folds without defined pathology excluding somatic dysfunction of less than 30 days duration. The author suggests exclusion criteria as follows: contraindications to OMT, pregnancy, neurodegenerative disease, prior surgery to the lumbosacral spine, history of chronic low back pain, and positive "red flags" for serious spinal pathology. The author also suggests that each institution would be obligated to assure an adequate sample size to more precisely address the research question.

In the suggested series of future research, the author also recommends that physical assessment should be standardized for each of the studies. The structural examination would use a standardized protocol to assess for postural imbalance, psoas dysfunction, quadratus lumborum dysfunction, piriformis dysfunction, sacral dysfunction, pelvic dysfunction, and lumbar segmental dysfunction. Each dysfunction would be treated using only the intervention modality, active control modality, or sham technique as defined by randomized group allocation. Further, the author recommends several measures to mitigate bias. A validated method of randomization must be utilized to assign group allocation; group allocation should be concealed from investigators and participants. Groups should be comparable at baseline, including major confounding factors, such as age, sex, body mass index, smoking status, employment status, fear-avoidant behaviors, and catastrophization. Comparison groups should receive the same care apart from the intervention being studied. Guideline-based conventional care should be the same for all studies. The accepted definition of conventional care is to explain the usual course of ANLBP, advise remaining active, and provide a non-steroidal anti-inflammatory drug or acetaminophen if indicated [3]. The number and the characteristics of dropouts for each group should be noted. Future investigators should, in the authors' opinion, assure an appropriate length of follow up, with intervention and control groups followed for an equal length of time; for example, outcomes measures may be obtained at baseline, two weeks, one month, and three months. Pain beyond three months would be considered chronic. This future study series could use self-reported pain and function ability as a definition of outcome; VAS and the Roland-Morris disability questionnaire [28] would be valid and reliable methods to determine the outcomes.

In this future study series suggested by the author, statisticians should be kept blind to the participants' group allocation and to other important confounders. Statistical assessment would be performed to assess the difference in the means of the outcome measures. The results should be 
reported with the corresponding standard deviations or confidence intervals. Finally, each study would be submitted for publication no matter the outcome, to avoid publication bias in a future MTC. The results of each study would then be used to generate a MTC of osteopathic techniques for the treatment of ANLBP. The resulting model would be at extremely low risk for within-study bias, reporting bias, indirectness, imprecision, heterogeneity and incoherence. Data gathered relating to specific dysfunctions and group characteristics could also be used for network metaregression to determine how a covariate might interact with the treatment effects.

There are many benefits to be gained by using this future study design recommended by the author, beyond efficiency. The final product would provide level $1 \mathrm{~A}$ evidence [29] comparing a variety of OMT techniques used for treating ANLBP. The acquired knowledge could then be applied to the development of clinical practice guidelines for the treatment of ANLBP. A study plan like this one would provide structure and training opportunities for institutions with fledgling clinical research programs. This approach to research is also aligned with the AOA's strategic plans for research [30].

\section{Conclusions}

This study failed to provide evidence to recommend for or against any of the included OMT techniques or prescribed exercises to conventional care for ANLBP. This may reflect the limited number of previously-published studies meeting inclusion criteria due to methodological heterogeneity, failure to adequately report results, and unacceptable risk of biases. However, the model's findings do align with some prior literature, suggesting that NMA for MTC of osteopathic techniques may be a useful method for examining some of osteopathic medicine's more complex questions if the shortcomings in osteopathic research are addressed in future investigations.

Research funding: None reported.

Author contributions: The author has responsibility for the entire content of this manuscript and approved its submission.

Competing interests: None reported.

\section{References}

1. Keeney B, Turner J, Fulton-Kehoe D, Wickizer TM, Chan KC, Franklin GM, et al. Early predictors of occupational back re-injury: results from a prospective study of workers in Washington State. Spine 2013;38:178-87.
2. Back injuries prominent in work-related musculoskeletal disorder cases in 2016. TED: the Economics Daily. U.S. Bureau of Labor Statistics website; 2018. https://www.bls.gov/opub/ted/ 2018/back-injuries-prominent-in-work-related-musculoskeletaldisorder-cases-in-2016.htm [Accessed 15 July 2020].

3. Paige NM, Miake-Lye IM, Booth MS, Beroes JM, Mardian AS, Dougherty $\mathrm{P}$, et al. Association of spinal manipulative therapy with clinical benefit and harm for acute low back pain: systematic review and meta-analysis. J Am Med Assoc 2017;317: 1451-60.

4. Qaseem A, Wilt T, McLean R, Forciea M. Noninvasive treatments for acute, subacute, and chronic low back pain: a clinical practice guideline from the American College of Physicians. Ann Intern Med 2017;166:514-30.

5. Liberati A, Altman DG, Tetzlaff J, Mulrow C, Gøtzsche PC, loannidis JP, et al. The PRISMA statement for reporting systematic reviews and meta-analyses of studies that evaluate health care interventions: explanation and elaboration. J Clin Epidemiol 2009;62:e1-34.

6. Matters G. A practical tool for searching health-related grey literature. cadth.ca https://www.cadth.ca/resources/findingevidence/grey-matters [Accessed 25 Jan 2021].

7. NICE. The guidelines manual. National Institute for Health and Care Excellence website; 2009 http://wwwnice.org.uk/ guidelinesmanual [Accessed 15 Jan 2020].

8. van Valkenhoef G, Lu G, de Brock B, Hillege H, Ades A, Welton N. Automating network meta-analysis. Res Synth Methods 2012;3: 285-99.

9. Bayesian evidence synthesis. gemtc.drugis.org website. https://gemtc.drugis.org/signin.html [Accessed 14 Jan 2020].

10. van Valkenhoef G, Bujkiewicz S, Efthimiou O, Reid D, Stroomberg C, de Keijser J. GeMTC manual. https://gemtc. drugis.org/manual.html\#heterogeneity [Accessed 14 Jan 2020].

11. Ali M, Selim M, Elwardany S, Elbehary N, Helmy A. Osteopathic manual therapy versus traditional exercises in the treatment of mechanical low back pain. Am J Med Med Sci 2015; 5:63-72.

12. Blomberg S, Svärdsudd K, Mildenberger F. A controlled, multicentre trial of manual therapy in low-back pain initial status, sick-leave and pain score during follow-up. Scand J Prim Health Care 1992;10:170-8.

13. Cruser D, Maurer D, Hensel K, Brown S, White K, Stoll S. A randomized, controlled trial of osteopathic manipulative treatment for acute low back pain in active duty military personnel. J Man Manip Ther 2012;20:5-15.

14. Goertz C, Long C, Hondras M, Lawrence D, Meeker W. Adding chiropractic to standard medical therapy for nonspecific low back pain. Spine 2013;38:627-34.

15. Hurley DA, McDonough SM, Dempster M, Moore AP, Baxter GD. A randomized clinical trial of manipulative therapy and interferential therapy for acute low back pain. Spine 2004;29: 2207-16.

16. Lewis C, Souvlis T, Sterling M. Strain-counterstrain therapy combined with exercise is not more effective than exercise alone on pain and disability in people with acute low back pain: a randomised trial. J Physiother 2011;57:91-8.

17. Machado L, Maher C, Herbert R, Clare H, McAuley J. The effectiveness of the McKenzie method in addition to first-line care 
for acute low back pain: a randomized controlled trial. BMC Med 2010;8:1-10.

18. Paatelma M, Kilpikoski S, Simonen R, Heinonen A, Alen M, Videman T. Orthopaedic manual therapy, McKenzie method or advice only for low back pain in working adults: a randomized controlled trial with one-year follow-up. J Rehabil Med 2008;40: 858-63.

19. Prashant N, Anand H, Subhash K. Comparison of muscle energy technique and positional release therapy in acute low back painRCT. Indian J Physiother Occup Ther 2010;4:32-6.

20. Saratchandran R, Desai S. Myofascial release as an adjunct to conventional occupational therapy in mechanical low back pain. Indian J Occup Ther 2013;45:3-7.

21. Schneider M, Haas M, Glick R, Stevans J, Landsittel D. A comparison of spinal manipulation methods and usual medical care for acute and sub-acute low back pain: a randomized clinical trial. Spine 2015;40:209-14.

22. Takamoto K, Bito I, Urakawa S, Sakai S, Kigawa M, Ono T, et al. Effects of compression at myofascial trigger points in patients with acute low back pain: a randomized controlled trial. Eur J Pain 2015;19:1186-96.

23. Vohra S, Jaiswal V. Effectiveness of strain counterstrain technique on quadratus lumborum trigger point in low back pain. IOSR J Sports Phys Educ 2014;1:53-8.
24. von Heymann W, Schloemer P, Timm J, Muehlbauer B. Spinal highvelocity low amplitude manipulation in acute nonspecific low back pain: a double-blinded randomized controlled trial in comparison with diclofenac and placebo. Spine 2013;38:540-8.

25. Salanti G, Del Giovane C, Chaimani A, Caldwell D, Higgins J. Evaluating the quality of evidence from a network meta-analysis. PloS One 2014;9:e99682.

26. Franke H, Fryer G, Ostelo R, Kamper S. Muscle energy technique for non-specific low-back pain. A Cochrane systematic review. Int J Osteopath Med 2016;20:41-52.

27. Hayden J, Van Tulder M, Malmivaara A, Koes B. Exercise therapy for treatment of non-specific low back pain. Cochrane Database Syst Rev 2005;3:CD000335.

28. Roland M, Morris R. A study of the natural history of back pain, part I: development of a reliable and sensitive measure of disability in low-back pain. Spine 1983;8:141-4.

29. Oxford Centre for Evidence-Based Medicine. Levels of evidence. Centre For Evidence-Based Medicine, University of Oxford. https://www.cebm.ox.ac.uk/resources/levels-of-evidence/ oxford-centre-for-evidence-based-medicine-levels-of-evidencemarch-2009 [Accessed 25 Jan 2021].

30. Degenhardt BF, Standley PR. Strategic plan for research: a role for everyone in promoting research in the osteopathic medical profession. JAOA 2013;113:654-9. 